\title{
Imminent understanding of the structure of nearby AGN from IFUs
}

\author{
Richard Davies
}

Max-Planck-Institut fur extraterrestrische Physik, Garching, Germany

Email: davies@mpe.mpg.de

\begin{abstract}
Integral field spectroscopy provides us with immensely rich datasets about spatially resolved distributions and kinematics of emission and absorption lines. In this contribution I will describe some of the key insights that have been made about AGN using optical, near infrared, and far infrared IFUs. These encompass gas inflow and outflow mechanisms, and the relations between star formation, the torus, and accretion onto the black hole. Progress so far has largely relied on archetypal and small sets of objects. In the future, a more statistically robust approach will be required. I will end by discussing a number of issues that can easily confuse an emerging picture, and need to be borne in mind for such surveys.
\end{abstract}

